\title{
LAS TECNOLOGÍAS APLICADAS A LA EDUCACIÓN ESPECIAL INTEGRADORA: LA CONTRIBUCIÓN DEL SOFTWARE EDUCATIVO "HÉRCULES Y JILÓ”
}

\author{
AS TECNOLOGIAS APLICADAS À EDUCACÃO ESPECIAL \\ INTEGRADORA: A CONTRIBUICCÃO DO SOFTWARE \\ EDUCATIVO "HÉRCULES E JILÓ"
}

\section{THE TECHNOLOGIES OF INFORMATION AND COMMUNICATION APPLIED TO INTEGRATING SPECIAL EDUCATION: THE CONTRIBUTION OF THE EDUCATIONAL SOFTWARE "HÉRCULES AND JILÓ”}

LES TECHNOLOGIES DE L'INFORMATION ET DE LA COMMUNICATION APPLIQUÉES À L'ÉDUCATION SPÉCIALE INTÉGRATIVE : LE LOGICIEL ÉDUCATIF “HÉRCULES ET JILÓ”

\author{
Catalina Maria Alonso * \\ Amaralina Miranda e Souza **
}

\section{RESUMEN}

Las Tecnologias de la Información y de la Comunicación resultan grandes aliadas del profesor para favorecer el proceso de enseñanza-aprendizaje, ya que posibilitan atender a diferentes formas de aprender y considerar las diferencias individuales de alumnos y profesores. El énfasis se debe dar a la adecuación de los medios utilizados para poder alcanzar los objetivos de aprendizaje, respetando las condiciones de respuesta de los alumnos y la metodología del profesor. Presentamos las lineas fundamentales que han servido de base en el diseño, la elaboración y aplicación del software educativo "Hércules y Jiló", recomendado para el trabajo pedagógico con alumnos que presentan necesidades educativas especiales. En este proceso se han abordado varias lineas de acción: 1. fundamentar su realización con una base científica y tecnológica, para lograr una relación y motivación amigable del alumno con la máquina; 2. ofrecer una base científico-pedagógicametodológica para intentar alcanzar un atractivo tal que el alumno pueda construir conocimiento a través de su interacción con el software y sus actividades derivadas. Se aspira a que las tecnologías, y en particular el software educativo, puedan contribuir a posibilitar el aprendizaje de todos los alumnos, que presenten o no necesidades educativas especiales.

Palabras-clave: Tecnologias de la información y de la comunicación. Software educativo. Educación especial.

* Profesora Doctora del Departamento de Didáctica Org. Escolar y DD EE - Facultad de Educación - Universidad Nacional de Educación a Distancia - UNED, Madrid - España. Investigadora premiada en el área de Estilos de Aprendizaje, Informática Educativa con varias publicaciones en estas áreas (calonso@edu.uned.es).

** Profesora Doctora del Departamento de Teoría e Fundamentos - Área de Educación Especial - Facultad de Educación -Universidade de Brasilia - Brasil. Investigadora en el área de Informática Aplicada a la Educación Especial / Inclusiva. Texto escrito a partir de mi Tesis Doctoral, dirigida por la profesora Catalina Maria Alonso, aprobada, Cum Laude, en la Facultad de Educación de la Universidad Nacional de Educación a Distancia - UNED - Madrid, día 20 de noviembre de 2006 (amara@unb.br). 


\section{INTRODUCCIÓN}

Actualmente, vivimos en un mundo globalizado, que está en constante transformación, donde cada día se crean nuevos recursos tecnológicos cada vez más desarrollados y modernos.

Al hacer hincapié en el hecho de que la escuela no puede existir sin una conexión con el mundo, el profesor no puede ignorar los recursos tecnológicos existentes, sino enriquecer su práctica utilizándolos en el proceso de enseñanza-aprendizaje, pues educar es desarrollar, lo más plenamente posible, todas las potencialidades intelectuales, afectivas, creativas (artísticas) y morales del individuo.

La tecnología mediática revolucionó el mundo de las comunicaciones y de las informaciones. Sin darnos cuenta, adaptamos nuestra forma de actuar, de pensar y de comunicarnos, por la integración de esos nuevos medios, a nuestros comportamientos. Dentro de este contexto, las alteraciones provocadas por las intermediaciones tecnológicas son innumerables: desde el teléfono al fax; del teléfono móvil al correo electrónico; de la televisión vía satélite a la internet. Vivimos otro estilo de vida y no siempre conseguimos darnos cuenta de ello.

El profesor necesita ejecutar su capacidad de improvisación de administrar situaciones inesperadas que exigen habilidad, creatividad y eficacia, a fin de que su intervención sea operante y eficiente, ya que como docente tiene un importante papel dentro del proceso de integrar los recursos tecnológicos en clase. La intervención del profesor es necesaria pues los medios de comunicación, por si solos, la mayoría de las veces, no consiguen una interacción entre el conocimiento propuesto y el nuevo saber.

Las tecnologías utilizadas en la educación constituyen, hoy día, un instrumento de enorme potencial para el enriquecimiento curricular y para una mejora de la calidad de la enseñanza.

La televisión, el vídeo, la radio y el ordenador constituyen importantes instrumentos pedagógicos auxiliares, sin que deban sustituir, por ello, las relaciones de comunicación e interacción directa entre el educador y el educando.

Una de las contribuciones de las tecnologías es la de favorecer el trabajo del profesor, enriqueciendo y diversificando su forma de conducir el proceso de enseñanza y de aprendizaje. Es necesario observar la complejidad de ese proceso, escogiendo como foco de análisis la producción y evaluación de materiales pedagógicos para ambientes de aprendizaje, enriquecidos por el uso de las nuevas tecnologías.

\section{EL PROFESOR Y LA MEDIACIÓN CON LAS TECNOLOGÍAS EN EL AULA}

La educación tecnológica promueve el despertar para la interpretación del contexto social actual a la luz de sus condicionamientos y fundamentos. Por eso es importante verificar cuestiones relativas a los valores del momento actual, haciendo especial hincapié en la dimensión ética en un mundo repleto de tecnología en todos los sectores.

Estas reflexiones nos remiten a la interpretación del mundo desde las Tecnologías de la Información y de la Comunicación, con el propósito de construir una educación 
transformadora que vaya más allá del espacio escolar, pues de todos es sabido que en los días actuales debemos integrar las dimensiones del saber-hacer al saber-ser. Para ello, necesitamos comprender y creer en una educación crítica que pueda estar atenta a los fundamentos teóricos del conocimiento y a la práctica social. Educación es esa mezcla de responsabilidad y de mucha esperanza en la posibilidad de transformaciones en la sociedad (GRINSPUN, 1999, p. 64).

La integración de las innovaciones tecnológicas a los procesos educativos dependerá de la concepción de la educación presente en las nuevas generaciones, que fundamenta las acciones y políticas del sector. Si creemos que la educación es un medio de emancipación (y no solamente un instrumento de dominación y reproducción de las desigualdades sociales), tendremos que defender una integración creativa, y no simplemente relativa al mercado, de estas tecnologías de comunicación que tanto nos preocupan como educadores, y nos solicitan como ciudadanos y consumidores.

En la actualidad es necesario que la escuela desarrolle una educación que consiga atender a los nuevos procedimientos de información y comunicación, propiciándoles a los alumnos medios apropiados para la construcción de sus conocimientos, sin olvidar su formación en términos de sentir y crear.

El gran desafío es la transformación de profesores capaces de tratar con alumnos algunas veces en situaciones extremas: con los alumnos que ya poseen conocimientos tecnológicamente avanzados y acceso pleno al universo de informaciones disponibles en los múltiples espacios virtuales; con los alumnos que se encuentran en plena exclusión tecnológica sin oportunidades para vivir y aprender en esta nueva realidad; y con las instituciones de enseñanza equipadas precariamente y con recursos mínimos para poder desarrollar su trabajo.

El ordenador en el aula permite al profesor atender a la diversidad de sus alumnos de forma individualizada, además de promover actividades colectivas que favorezcan la cooperación entre ellos, resultando una práctica inclusiva e integradora, donde todos estarán actuando y aprendiendo entre ellos.

Para que se puedan realizar esas y otras transformaciones esperadas en la enseñanza, es necesario que el profesor sepa tratar críticamente las tecnologías contemporáneas de información y comunicación y que sepa utilizarlas pedagógicamente. El profesor debe conocer el ordenador, los soportes mediáticos y todas sus posibilidades educativas e interactivas de las redes y espacios virtuales para aprovecharlas en las más diversas situaciones de aprendizaje y en las más variadas realidades educativas.

Se debe trabajar satisfactoriamente con el conocimiento ya adquirido y a la búsqueda de nuevas informaciones, con los cambios estructurales de los saberes en todas las áreas, con una investigación permanente. Y también asumir una postura de inquisición, crítica y duda ante las informaciones - recientes y viejas - y, al mismo tiempo, ejercer el papel de orientación y cooperación con los alumnos. Enseñarlos a aprender, y aprender enseñando.

El profesor necesita del uso de diferentes lenguajes para sobrevivir en el mundo del trabajo actual. Múltiples lenguajes, que van desde la influencia tecnológica al dominio de idiomas, para que pueda salir del cerco de la clase y del ambiente escolar para conectarse, 
junto a sus alumnos, con todo el mundo, promover las relaciones institucionales con otros profesores, de otras escuelas o países, con distintas culturas y realidades sociales. El principal valor, la autoestima como profesor, esto es, ser profesor con orgullo y por opción.

Además, el profesor, debe identificarse con su función, con su estilo y con su propia profesión. El profesor debe tener capacidad de liderar y cooperar, de respetar las diferencias, de participar, de atreverse, de ir más allá, de aceptar desafíos y buscar su permanente actualización, poseer autonomía para enseñar y para aprender y, especialmente, para ser profesor, conocerse como persona y ser consciente de sus límites y avances.

Todos estos comportamientos desafiantes, provenientes de la integración de las nuevas tecnologías digitales al ambiente educativo, imponen cambios estructurales a la acción docente y a las formas de enseñanza. Se exigen nuevas posturas que posibiliten a los profesores la soltura metodológica adecuada para realizar una enseñanza de calidad, aunque las propias concepciones de lo que representa la enseñanza y de lo que es el aprendizaje se hayan transformado.

Ante todo, es necesario que exista cierta transparencia y claridad sobre las reales posibilidades de acción del docente con el uso de las tecnologías. Una educación crítica sobre los medios de comunicación, retirándoles el aura de "cajas-negras", objetos mágicos poderosos y temidos, situándolos en sus debidos lugares, como equipos, herramientas y espacios - en este caso, educativos - que pueden revolucionar la enseñanza y auxiliar a los profesores y alumnos a enseñar y a aprender.

Este profesor que circula libremente en el medio digital encuentra allí un espacio educativo radicalmente diferente. Comprende que su acción docente en este nuevo ambiente apenas requiere un cambio metodológico, sino un cambio de percepción de lo que es enseñar y aprender. Objetos multimedia digitalizados - sonidos, imágenes y texto pueden ser creados, editados, integrados y transmitidos con gran facilidad. Instructores y estudiantes pueden controlar sus presentaciones y análisis con un poco más de experiencia en ordenadores, más allá de las posibilidades del uso del teclado y del ratón.

Las posibilidades interactivas del ambiente digital involucran a estudiantes y profesores en el aprendizaje. El aprendiz puede llegar a tener mayor responsabilidad por conducir el proceso educativo y aprender a utilizar herramientas, entre otras, procesadores de textos, planillas, que serán útiles en el mundo real.

Por medio de la tecnología se pueden realizar actividades integradas y potenciar la búsqueda de informaciones sobre lo cotidiano, que ayuden a la aprehensión del conocimiento científico, siempre que el profesor sepa articular teoría y práctica. En realidad, esa cuestión es el motivo de preocupación cuando se conoce que muchas escuelas aún no reúnen las mínimas condiciones de infraestructura para la realización de sus actividades en la enseñanza. Sin embargo, se observa que los espacios de investigación en las escuelas se han visto multiplicados, a medida que los profesores recurren a la creatividad para acceder a las informaciones, bien de forma directa o indirecta.

Por ello, podemos servirnos de las tecnologías y de los medios de comunicación para incrementar el trabajo realizado en clase, pues una de las grandes preocupaciones que ronda actualmente el campo educacional es la reducción de la distancia entre el mundo 
real y el mundo virtual. La escuela es responsable de conseguir un aprendizaje articulado con lo cotidiano, permitiendo una enseñanza con un mínimo de condiciones que favorezca el entusiasmo y el interés en la búsqueda del conocimiento científico.

\section{EL PROFESOR FACILITADOR EN LA CONSTRUCCIÓN DEL CONOCIMIENTO}

A partir del principio de que el alumno debe participar activamente en la producción del conocimiento, no podemos olvidarnos que hoy tenemos como aliados del trabajo escolar una inmensa cantidad de materiales tecnológicos que, a su vez, permiten que el alumno haga, construya y organice su propio saber.

Preocupados por permitir que el alumno haga y construya, el profesor debe repensar una nueva didáctica y diseñar un nuevo tipo de aprendizaje, con el propósito de favorecer al alumno en la búsqueda del conocimiento. En este mundo actual, se observa que el avance tecnológico en los medios de información y comunicación funciona a ritmo acelerado. Nos encontramos, entonces, ante una situación "sui generis" en lo referente a los aspectos didáctico-pedagógicos.

La búsqueda de esa nueva didáctica implica particularmente una reflexión sobre el compromiso en relación con el abordaje diversificado en la construcción del conocimiento, requiriendo una nueva interpretación de la unicidad teoría-práctica en el aspecto pedagógico. Dicha unicidad, a su vez, demanda transformar los elementos didácticopedagógicos: contenido / método, relación profesor / alumnos, planeamiento / ejecución, fines / evaluación (ALONSO, 2001).

Para ello, es necesario reflexionar sobre el papel del profesor. Como mediador, orientador o facilitador; el profesor siempre es motivo de discusión en lo referente a las formas de organización innovadoras del proceso pedagógico, principalmente cuando se le atribuye a la escuela la característica de un espacio autónomo, cuya configuración y funcionamiento tienen como elemento decisivo la acción e interacción de los diferentes actores (profesores, dirigentes, funcionarios, alumnos y padres).

En un ambiente de estudio con disponibilidad de recursos tecnológicos, ya sea informatizado o no, el profesor debe proporcionar condiciones para un aprendizaje significativo, promoviendo la construcción del conocimiento, a través de la reinvención. Para que el alumno pueda construir el conocimiento es necesario propiciar ambientes que lo desafíen y lo motiven para la exploración, reflexión, depuración y descubrimiento de ideas. Y el profesor debe ser creativo, buscar alternativas entre los recursos tecnológicos disponibles y ofrecer al alumno el soporte necesario para ayudarle en el procesamiento de informaciones. Asimismo, al docente le corresponde valorar la iniciativa del alumno, interviniendo en actividades que no sean meras secuencias sistematizadas ni tampoco simples experimentos espontáneos. En esos ambientes, las actividades se desarrollan alrededor de proyectos y no se atienen a contenidos específicos previamente establecidos. 
La utilización de los recursos computacionales debe alertar al docente de que, para un aprendizaje significativo, el alumno debe ser el sujeto de su propia acción, participar activamente en un proceso continuo de colaboración, motivación, investigación, reflexión, ganando autonomía e independencia.

Las herramientas tecnológicas no auxilian sólo en la articulación entre el conocimiento y la mente, promoviendo movimientos sociales, culturales e intelectuales, sino también contribuyendo para el aprendizaje significativo sobre el tema propuesto. Por ello, el foco central de los estudios de Papert $(1981,1995)$ no es la máquina, sino la mente. El aprendizaje del alumno sólo se produce cuando actúa sobre los contenidos específicos y en la medida en que posee estructuras propias, previamente construidas o en construcción (ALMEIDA, 2001, p. 61).

Para Papert (1995), es necesaria una metodología que nos permita permanecer cerca de situaciones concretas, pues el hacer y el comprender están vinculados a los problemas con los que el individuo se enfrenta en su realidad física o social. Esa idea se aproxima a la teoría de Vigotski (1994), según la cual el aprendizaje se encuentra en el desarrollo histórico-social del sujeto.

En el contexto de la práctica pedagógica cotidiana, el ordenador podrá ser utilizado por el profesor como un importante instrumento pedagógico. Sabiendo explorar esa herramienta y trabajar con proyectos que surgirán en clase, el educador podrá proporcionar un aprendizaje en este enfoque, contextualizado y significativo. Sin embargo, el uso del ordenador no podrá ser privado de las relaciones humanas, ya que la construcción del saber depende, sobre todo, de la interacción profesor-alumno-ordenador, pues el profesor es pieza clave en el desarrollo de las habilidades, el lado afectivo y los valores de cada alumno.

Al hablar sobre el uso de tecnologías como recursos didácticos, es importante recordar el proceso de interacciones entre el profesor y los alumnos, puesto que este proceso [...] contribuye, con naturalidad, a la construcción del conocimiento, pues debe suceder con amplia participación del alumno que utiliza sus experiencias sociales y de vida como autor de sus propios caminos (SILVA, 2004, p. 245). Entre esas interacciones, destacamos el hecho del profesor actuando como mediador además de trabajar con actividades lúdicas. Todos estos aspectos facilitan una mayor aproximación entre alumno y profesor, pues éste es un camino de doble dirección: un proceso de intercambios que favorece en el profesor aprender con el alumno, aprender y enseñar, enseñar y aprender.

A ese respecto, Lévy (2001, p. 30) nos recuerda que:

[...] la escuela debe convertirse en un lugar de inteligencia colectiva, de cooperación, de apertura, de discusión entre las asignaturas y especialidades, de circulación de las personas y de los saberes. Ella debe recompensar la originalidad, la curiosidad, lo desconocido. Sólo se aprende aquello que se practica, lo que es ejercitado con frecuencia, lo que está integrado en los reflejos. 


\section{LA INFORMÁTICA EDUCATIVAY LA ATENCIÓN A LA DIVERSIDAD}

El ordenador resulta un instrumento utilizable por su "multi-funcionalidad", para realizar varias tareas, lo que ofrece nuevas posibilidades permitiendo que la misma máquina sea utilizada para fines y actividades diferentes. Esa multifuncionalidad también facilita atender a las diferencias individuales de los usuarios. Así, el ordenador puede ser ajustado a las características y necesidades de las personas.

En el ámbito escolar, el uso pedagógico del ordenador se considera como un factor de avance cualitativo del proceso enseñanza-aprendizaje.

Además, existe la idea central de que la informática educativa puede volverse un poderoso aliado del profesor en el proceso de facilitar la construcción del conocimiento; el profesor puede servirse de ella para crear situaciones de aprendizaje bastante motivadoras y productivas. La introducción del ordenador en nuestras escuelas, por ejemplo, está planteando nuevas formas de trabajo por tratarse de un medio que puede ser utilizado como recurso educativo muy poderoso y versátil.

Según Valente (1991, p. 29) el uso del ordenador en el aula, como medio educativo, ha cuestionado los métodos y procesos de enseñanza utilizados, y ha posibilitado entender que el profesor puede ejercer otras funciones, además del mero papel de transmisor del conocimiento. También puede facilitar el aprendizaje, algo que con el ordenador se consigue también.

Sin duda existe una crítica al uso del ordenador simplemente como el de una herramienta de ejecución de programas automatizados que sirve a objetivos muy limitados y no permite al alumno realizar un aprendizaje más profundo. Aquellos que defienden la idea de introducir en su programa el uso del ordenador como parte del currículum, recomiendan lenguajes más simples, de forma que el ordenador funcione como un recurso, un medio con el cual el alumno desarrolle su aprendizaje en algunos aspectos más complejos.

Es decir, el ordenador como herramienta presenta ciertas características que resultan de gran contribución, especialmente al ser utilizado como recurso educativo (ESPAÑA, 1992), porque:

- Se trata de un medio motivador para el niño y posibilita incluir en los programas música, colores, animación. Así se convierte en material con atractivos que contribuyen a centrar la atención.

- Tiene una gran capacidad de memoria, pudiendo almacenar un amplísimo número de datos y permite una cierta rapidez en la ejecución de las tareas.

- Presenta una gran versatilidad, permitiendo ser utilizado en muy distintos ámbitos y con finalidades muy diversas, lo que responde a la filosofía de seleccionar materiales que puedan ser adaptables a las distintas necesidades de los alumnos.

- Puede ser una herramienta con gran potencial para reforzar estrategias de resolución de problemas ofreciendo distintas oportunidades para resolverlos, haciendo más hincapié en los éxitos que en los fracasos, lo que puede ayudar a disminuir la frustración ante el error. 
- Posibilita plantear situaciones de trabajo de grupo, con programas que admiten interacción de varios niños, dando lugar a situaciones de trabajo cooperativo y diálogo espontáneo que influyen positivamente en el proceso de socialización.

- Se adapta a las peculiaridades de cada alumno, respetando su ritmo de aprendizaje. En este caso el ordenador se caracteriza, entre otras cosas, por tener una paciencia infinita, pudiendo repetir una tarea tantas veces como el usuario desee y ejecutando sólo aquello que se ha pedido, sin tomar ninguna decisión por su cuenta.

Además de como herramienta, el ordenador puede ser adaptado a los diferentes estilos de aprender, a los diferentes niveles de capacidad e interés intelectual, a las diferentes situaciones de enseñanza - aprendizaje, posibilita crear nuevas maneras de enseñar que puede implicar cambios que quizá el sistema escolar aún no esté preparado para realizar con flexibilidad. Por todo esto hay que determinar cómo podemos incorporar el ordenador como una herramienta potencialmente útil, de manera que se integre en el aula, en el currículum y que permita al profesor ajustar su trabajo en la clase para ofrecer al alumno diversas posibilidades de respuestas educativas cada vez más positivas.

\section{LA INFORMÁTICA EDUCATIVA APLICADA A LA EDUCACIÓN ESPECIAL INTEGRADORA/ INCLUSIVA}

El descubrimiento de nuevas formas de enseñar y aprender por medio de la informática educativa es un desafío extremamente motivador, que implica y que demanda trabajos de investigación dirigidos a la producción de medios y materiales que posibiliten también la teoría respecto de su aplicación en la relación mediada por esta tecnología.

Existen varias investigaciones que revelan que la informática tiene potencial para dinamizar el proceso enseñanza-aprendizaje. En particular el software educativo, cuando es adecuadamente empleado, establece relaciones cualitativamente diferentes y puede funcionar como instrumento de liberación de la situación pedagógica, proporcionando un espacio de libre descubrimiento, aprendizaje constructivista, para realimentar y redimensionar la práctica pedagógica del profesor para hacerla más significativa (SANTOS, 2000).

Se destaca también que la atracción ejercida sobre los alumnos al usar el ordenador, la rapidez en la realización de las tareas, el respeto al ritmo de aprendizaje del alumno, la diversificación de las rutas que llevan a la resolución de problemas, la posibilidad de trabajo colectivo, entre otros aspectos, hacen pensar en las ventajas que se pueden obtener con el uso correcto del ordenador para objetivos educacionales.

Martí (1992), citado por Pérez (2003, p. 431), comenta algunos inconvenientes en el uso de la informática con los alumnos con discapacidad, sobre todo por la rapidez de su evolución que puede dejar obsoletos algunos productos, sin olvidar que, en general, son muy pocos los programas elaborados para atender a las singularidades de los alumnos con necesidades educativas especiales. No obstante considera que, pese a todo, el empleo de la informática para los alumnos con discapacidad es de gran utilidad porque puede contemplar algunos aspectos que consigue el ordenador, entre los que destaca: 
- Posibilita una mejora en las capacidades de comunicación, con un mejor control sobre su entorno, y potencia la autonomía;

- Ayuda a mantener el interés para continuar realizando la actividad propuesta porque con el ordenador la persona con discapacidad domina más el entorno, además aumenta la autoestima porque al acceder a actividades más complejas, que hasta ahora no podía realizar, aumenta la confianza en sí misma e incentiva a seguir en búsqueda de otros aprendizajes;

- Permite aprender estrategias cognitivas adecuadas para solucionar problemas, de manera que consiga tener un mayor control sobre el propio entorno del aprendizaje. Especialmente importante para los alumnos que presentan deficiencia intelectual, cuyas habilidades meta-cognitivas están claramente afectadas.

López (1995, p. 582) atribuye a la aplicación de las tecnologías en la Educación Especial, específicamente con los medios informáticos, muchas ventajas:

- La versatilidad y flexibilidad que permiten múltiples aplicaciones con objetivos diversos y adaptaciones en casos particulares.

- Facilidad de individualización de la enseñanza, adecuando las tareas al nivel de competencias del alumnado y de acuerdo con su propio ritmo de aprendizaje.

- Posibilidad de repetición del ejercicio y la auto-corrección, al poder comprobar los resultados de inmediato.

- Aumenta el grado de autonomía e independencia personal y permite al profesor disponer de más tiempo de dedicación a otros alumnos que lo necesiten más.

En este sentido, existen datos de que trabajos realizados con los alumnos con necesidades especiales muestran que el ordenador puede ayudarles a adquirir aprendizajes importantes, posibilitándoles a enriquecer sus capacidades intelectuales, su sentido de autoestima para, de manera independiente, explorar y ejercitar sus propias acciones.

Por otra parte, es grande el desafío al intentar crear ambientes de aprendizaje con la perspectiva de construcción de conocimientos utilizando el ordenador. Esto implica considerar los cambios de comprensión acerca del ordenador como una forma de representar el conocimiento: el significado de enseñar y aprender y el papel del profesor en este nuevo contexto (VALENTE, 2001).

\section{EL SOFTWARE EDUCATIVO COMO RECURSO DIDÁCTICO PEDAGÓGICO DE APOYO AL PROCESO ENSEÑANZA-APRENDIZAJE}

El uso de la informática en la educación exige un esfuerzo constante por parte de los educadores para transformar la simple utilización del ordenador en un abordaje educacional que favorezca efectivamente el proceso de adquisición de conocimiento por el alumno.

Para Valente (1991, p. 18), una de las cuestiones fundamentales en el desarrollo de software educativo es el aspecto pedagógico: qué es lo que el software se propone enseñar y cómo esto es realizado. 
Lo que caracteriza un software como educativo es su inserción en el contexto de enseñanza-aprendizaje, el hecho de ser desarrollado con la finalidad de llevar al alumno a construir determinado conocimiento relativo a un contenido didáctico.

En este contexto, el software educativo para la educación mediada por el ordenador tiene la importante función de permitir que el alumno se sirva de él para interactuar cognitivamente con el contenido disponible, construyendo nuevos conocimientos e instrumentos para transferirlos en otras situaciones, en su medio y en su vida cotidiana.

En el lenguaje LOGO, por ejemplo, el proceso de creación es más importante que el producto en sí. Además, el uso del lenguaje, en la forma expuesta por su autor, permite la caracterización y el seguimiento de los esquemas con los cuales el niño actúa en cada momento, lo que favorece conocer sus procesos cognitivos y por lo tanto un trabajo pedagógico a la altura de su zona de desarrollo proximal, descrita por Vigotski (2000).

Como en todo proceso de enseñanza aprendizaje, el papel del profesor en la mediación de las actividades de los alumnos es esencial e implica la creación de ambientes de aprendizaje creativos y motivadores.

Muchas veces la rutina de clase y las dificultades de los alumnos pueden hacer que el profesor no perciba que determinado alumno no sigue el ritmo de su grupo y evidencia bajo rendimiento escolar. Ante el ordenador, con una propuesta y un ambiente de aprendizaje diferente, el alumno "olvida", "se relaja" y puede revelar los conocimientos que realmente ya construyó.

O aún, otros niños de los que se preocupan los profesores, porque piensan que no aprenden nada, porque su vínculo con el aprendizaje formal está mal, parece que sí funcionan en un nivel cognitivo inferior al esperado para su grupo y, muchas veces, pueden revelar sus reales adquisiciones en las propuestas surgidas en el ambiente multimedia con el ordenador.

Para que estas situaciones puedan suceder, es necesario construir un puente entre las actividades curriculares y el uso del ordenador, integrando todo el trabajo, y concediéndole al niño con difícultades una mejor relación con el medio en el que vive, la oportunidad de auto-conocimiento. Esa vivencia despierta en el alumno la oportunidad de reflejar su desarrollo, y su seguridad ante situaciones desconocidas, además de llevarlo a reflexionar sobre su propio pensamiento (meta-cognición).

La meta-cognición permite que las diferencias individuales tengan relevancia en consideración al desarrollo cognitivo y en relación a la enseñanza y al aprendizaje (TARDIF, 1992).

Podemos observar que el uso del ordenador despierta la motivación en la mayoría de los alumnos y que esto puede ser una buena oportunidad para que el profesor rescate al alumno que no va bien en clase, y que pueda funcionar como un instrumento que permita una interacción alumno-objeto, alumno-alumno, y alumno-profesor, basado en los desafíos e intercambios de experiencias.

El profesor necesita plantear situaciones educacionales que promuevan el aprendizaje, llevando a los alumnos a la exposición de sus conocimientos y dudas, a llenar los 
espacios vacíos, a apropiarse de nuevos conocimientos. También es necesario orientar la interacción social por medio de reglas claras y bien definidas, organizadas, integrando y presentando los conocimientos, proponiendo actividades, ayudando a los alumnos a superar las dificultades que aparecerán a lo largo del saber (SCOZ, 1994).

Entre las variadas características que podemos citar para el software educativo existe una que, sin duda, refleja todo el esfuerzo de los elaboradores del material didáctico y que cumple los objetivos del sistema educativo: la transferencia de los conocimientos disponibles en el material didáctico a otras situaciones distanciadas de la relación educativa propiamente dicha.

En la literatura pedagógica la transferencia de los aprendizajes ocupa un lugar destacado. Varios pedagogos e investigadores discuten sobre las modalidades de enseñanza que mejor pueden favorecer la transferencia de conocimientos, y destacan que la escuela contemporánea aún no ha conseguido integrar estos mecanismos.

En el contexto de la educación especial, el concepto de transferencia de conocimiento es fundamental porque se adapta a las propias características de los alumnos con necesidades educativas especiales. El software educativo necesita estar orientado para que el alumno interactúe con el conocimiento que articula, y lo instrumentalice para reaplicar algunas situaciones en otras distintas, modificando su práctica, reafirmando su pertinencia y apoyándose definitivamente en su memoria a largo plazo.

$\mathrm{Al}$ mismo tiempo que facilita la comunicación entre el profesor y el alumno, el software educativo organiza las actividades de aprendizaje, de tal forma que las intervenciones del profesor se concretan en las acciones del alumno. Esto sucede porque, de una forma o de otra, su elaborador presenta una representación particular del conocimiento deseado.

La creación de un espacio libre para la interactividad cognitiva debe ser otra característica importante del software educativo. No obstante, aunque el alumno se distancie del proyecto comunicativo proporcionado por el software, el material didáctico tiene siempre que ofrecer espacio para que la interactividad sea potenciada al máximo, o sea, para que el alumno integre los contenidos propuestos y haga de ellos un proyecto de formación, llegando a extrapolar esos logros para avanzar en dirección complementaria o de profundización.

Por otra parte, se observa que la mayoría del software desarrollado para la educación especial parte del principio de que los niños con necesidades educativas especiales tienen baja capacidad para aprender y, por lo tanto, las actividades están niveladas con esa capacidad, y la pedagogía adoptada enfatiza la repetición de actividades poco complejas (SOUZA, 2005).

Se puede decir, además, que en el centro de estas dificultades están las dificultades del sistema educativo, principalmente en la escuela y en la precaria formación del profesor para actuar con la informática educativa, específicamente para integrar el ordenador como apoyo para el desarrollo de sus actividades con el alumno en clase.

Para Torres (1990), el ordenador también ofrece ventajas para trabajar con niños que presentan necesidades educativas especiales, porque ahorra tiempo y esfuerzo; introduce 
una dinámica visual importante; incrementa la motivación y refuerza la atención; prepara al alumno para el aprendizaje permanente y autónomo; contribuye a las capacidades de autoaprendizaje estimulando la creatividad; ofrece la posibilidad de una evaluación dinámica continua y objetiva; contribuye a la adquisición de capacidades básicas como son la lectura y escritura, la expresión de cálculo, el pensamiento lógico y la resolución de problemas.

Además, el uso del ordenador en clase puede ayudar al profesor a: organizar mejor su actividad educativa, evaluar permanentemente la situación del niño registrando los cambios producidos, observar el grado de aprendizaje de cada uno de los alumnos, identificar las diferencias y las necesidades para introducir nuevas estrategias que faciliten el proceso de aprendizaje.

El profesor puede sacar provecho del software educativo para comprender, criticar y hasta concebir su propio material didáctico, a partir del cual podrá obtener el avance cualitativo de su enseñanza, obteniendo provecho de las variadas posibilidades ofrecidas por la informática educativa.

\section{LA ENSEÑANZA Y EL APRENDIZAJE DEL ALUMNO CON NECESIDADES EDUCATIVAS ESPECIALES MEDIADOS POR EL ORDENADOR}

El aprendizaje mediado es un proceso interactivo a través del cual el profesor potencia la interiorización de los procesos psicológicos del pensamiento, por tanto el mediador ayuda al alumno a organizar, filtrar y esquematizar los estímulos de aprendizaje y finalmente influye en la trasferencia (PRIETO, 1996).

El profesor como mediador, éste debe poner en marcha cierta parte del potencial intelectual del alumno que no surge de forma espontánea y que es necesario trabajar intencionalmente. El profesor es quien facilita el aprendizaje, un investigador de los procesos acaecidos en el aula.

En el aprendizaje constructivo, el alumno logra relacionar la nueva tarea de aprendizaje con sus conocimientos y experiencias previas, almacenadas en su estructura cognoscitiva. De ahí que esas ideas y circunstancias son comprendidas y asimiladas significativamente durante su aprehensión. El alumno es un agente activo que procesa y hace categorías de la información del ambiente, interpretándola, dándole sentido y transfiriéndola a otras situaciones.

Con este nuevo escenario, por tanto, se hace necesario diseñar un sistema de aprendizaje, una nueva forma de aprender con tecnologías que esté en consonancia, por una parte, con la nueva concepción del aprendizaje y, por otra, con las necesidades de aprendizaje tecnológico de los sujetos con necesidades educativas especiales.

Es recomendable que los materiales informáticos permitan que el alumno haga actividades que favorezcan la interiorización de los contenidos para poner en práctica lo aprendido, y actividades de desarrollo cognitivo para reforzar los procesos de pensamiento implicados en el aprendizaje, a través de tareas que promuevan el desarrollo de la capacidad intelectual. 
Las actividades creadas deben potenciar los variados aspectos del desarrollo y del aprendizaje del alumno. Cabe al profesor realizar la exploración pedagógica más oportuna y adecuada para el alumno, para el contexto del aula y para los objetivos de aprendizaje que pretende alcanzar:

1. Debe potenciar la atención, la memoria, el proceso de generalización, la iniciativa para comenzar una actividad, la autonomía personal y disminuir el sentimiento de frustración. Todo ello integrado en el diseño de los juegos y actividades para crear situaciones y espacios variados para que el aprendizaje ocurra.

2. Debe privilegiar la interacción del alumno con el profesor, con el material, con sus colegas a través del ordenador, que relacionen las actividades y el contenido informatizado con nuevas posibilidades de exploración también fuera del ordenador.

Es necesario considerar que los alumnos con necesidades educativas especiales pueden aprender y que, a su vez, se van beneficiando de los desarrollos que se hagan para los demás alumnos. En este caso el ordenador, y muy especialmente el software educativo, puede ser aplicado considerando los aspectos de la motivación, de las dificultades y, sobre todo, de las capacidades que presentan, y que pueden constituir una posibilidad concreta para el desarrollo e integración social y escolar de estos alumnos.

Para el alumno con necesidades educativas especiales, el profesor que funciona como mediador favorece su proceso de aprendizaje porque pone en marcha cierta parte de su potencial intelectual, casi siempre ignorado. Esto no surge de forma espontánea y por eso necesita ser trabajado de forma intencional con planteamientos que utilicen recursos y estrategias pedagógicas dirigidas para que el aprendizaje acontezca.

\section{LA CONTRIBUCIÓN DEL SOFTWARE "HÉRCULESY JILÓ”: INTERROGANTES Y RESPUESTAS SOBRE LA APLICABILIDAD Y APOYO AL APRENDIZAJE DE ALUMNOS CON NECESIDADES EDUCATIVAS ESPECIALES}

El software educativo "Hércules y Jiló" fue ideado para servir de apoyo a las intervenciones pedagógicas en el área de Ciencias Naturales, tratando conceptos relacionados con los seres vivos que existen en la tierra (diversidad, características, clasificación, relaciones tróficas, ambientes naturales y ambientes construidos).

Concebido de acuerdo con principios cognitivos-constructivistas, según los cuales aprender es un proceso activo de establecimiento de puentes entre nuevas informaciones y conocimientos anteriores, que requiere una reorganización constante de conocimientos declarativos procedimentales y condicionantes, a partir del empleo de estrategias y metaestrategias referenciados por Pena (1984) y Tardif (1992),el software en referencia propone servir como apoyo al trabajo pedagógico del profesor considerando las características y necesidades educativas del alumno con deficiencia intelectual.

El software "Hércules y Jiló" pretende estimular la reacción del niño mediante recursos múltiples, buscando la interacción entre lo virtual y lo real, objetivando la alfabetización científica, matemática, lingüística, social e informática. Está basado en cinco dimensiones: de contenido, didáctica, cognitiva, lúdica e interactiva. Esas dimensiones son interdependientes 
y complementarias y se relacionan de manera dialéctica para que los objetivos de aprendizaje sean completamente realizados.

El funcionamiento del software educativo "Hércules y Jiló" está centrado en una serie de actividades, de forma aleatoria y gradual, para que el usuario debidamente orientado las puedan realizar. Esas actividades se dividen en dos categorías:

1. actividades para armar y jugar, en las que el niño utiliza el ordenador para construir juegos para jugar fuera del ordenador.

2. actividades interactivas y virtuales en las que el niño es estimulado a participar en una serie de juegos virtuales propuestos por el software. Todas las actividades abordan el tema de seres vivos que existen en la tierra.

El software "Hércules y Jiló", por sus características generales, se puede definir como un ambiente de aprendizaje multimedia.

Un ambiente de aprendizaje multimedia consiste en la asociación de recursos (materiales, informáticos, humanos, etc.) alrededor de un proceso dinámico de enseñanzaaprendizaje. Estos recursos están estratégicamente asociados para potenciar al máximo el acto de aprender, sea libremente con actividades lúdicas y pedagógicas, sea en situaciones de trabajo cooperativo (SOUZA; SANTOS, 2001).

La aplicación del software educativo "Hércules y Jiló" en una clase de enseñanza especial fijó el objetivo de observar para discutir la informática como apoyo al proceso enseñaza-aprendizaje del alumno con deficiencia intelectual (VERDUGO, 1997, p. 24), más específicamente su contribución como recurso de apoyo didáctico al trabajo pedagógico del profesor.

Se buscó observar en el trabajo de la profesora que lo implementaba, utilizando el software "Hércules y Jiló" como recurso didáctico pedagógico, con el tema del software (los seres vivos) en los juegos y actividades, la relación de la profesora con el software y con los alumnos en sala de aula, así como la relación de los alumnos con el ordenador y principalmente con el software y de éstos con la profesora, trabajando con el referido software.

Para complementar los datos recogidos a través de la observación realizada, fue hecha, al final, una entrevista semi-estructurada con la profesora buscando conocer sus impresiones sobre el uso del software educativo "Hércules y Jiló", como recurso pedagógico de apoyo a su trabajo con los alumnos.

Planteamos, ahora, la investigación realizada durante varios años sobre el diseño, la elaboración y sobre todo la evaluación del software "Hércules y Jiló" y su repercusión en la aplicación en el trabajo diario con alumnos con deficiencia intelectual. Presentamos la síntesis de todo ello en forma de interrogantes con sus respectivas respuestas, que pueden servir como orientación para el uso de software educativos como apoyo al proceso enseñanza-aprendizaje de estos alumnos.

Interrogante $1-¿ Q$ Qué estrategias debe presentar el software educativo para poder provocar, adecuadamente, aprendizajes significativos y pertinentes en el niño con deficiencia intelectual? 
El estudio hecho señala la necesidad del software de responder bien al tratamiento del contenido elegido, favoreciendo siempre la interacción con el público al que se destina. Es necesario también que las interfaces estén diseñadas y construidas para atender a los objetivos que se proponen, que implican considerar las características y necesidades de los usuarios.

Interrogante 2 - ¿Qué características puede presentar un software educativo para funcionar como apoyo al profesor en el proceso de enseñanza-aprendizaje del alumno con deficiencia intelectual?

En relación con el profesor, la experiencia observada en la aplicación del software destaca la importancia de que esté ambientado para que el profesor pueda conducir las actividades con los alumnos, de forma a no dejar al alumno solo en el ordenador, sino trabajar juntos. En este sentido, el software necesita garantizar mecanismos para que el profesor lo maneje con facilidad para tener un papel activo de mediador del aprendizaje con el alumno. Las actividades interactivas que conducen al trabajo colectivo favorecen el aprendizaje. Este aspecto fue muy enfatizado en la evaluación y aplicación del software analizado.

Observamos en la aplicación del software "Hércules y Jiló" que es muy importante que el software sea flexible y que pueda permitir la intervención didáctica del profesor y, a ser posible, pueda ser modificado por él, para atender a los objetivos de la enseñanza pero sobre todo para atender a las necesidades educativas del alumno.

Las teorías de aprendizajes elegidas necesitan ser claramente contempladas en las actividades propuestas, así como los principios pedagógicos con los cuales el profesor va a trabajar, para fundamentar y garantizar el proceso de enseñanza-aprendizaje. En nuestro caso, los principios del aprendizaje fueron claramente establecidos respetando las características del aprendizaje del alumno con deficiencia intelectual para favorecer el aprendizaje del contenido planteado. El software necesita posibilitar al profesor para que pueda identificar los avances del alumno. Debe permitir que el profesor pueda evaluar los progresos y dificultades del alumno para alimentar su acción pedagógica, y le permita ascender en su conocimiento y aprendizaje.

Interrogante 3 - ¿Desde qué forma el software educativo puede servir como factor de motivación para el niño con deficiencia intelectual en el proceso de adquisición de conocimiento?

Resultó interesante observar en la aplicación del software "Hércules y Jiló" cuán importante es potenciar las capacidades de los alumnos y de los profesores para promover la motivación. Las actividades propuestas necesitan ser desafiantes y adecuadas al nivel de interés del público y al mismo tiempo de fácil comprensión para que el usuario pueda utilizar las funciones cognitivas en una perspectiva de aprendizaje significativo.

Particularmente en este estudio observamos que los alumnos estaban motivados para realizar los juegos / actividades, aún sin conocer las respuestas o cuando tenían dificultades en el manejo del ordenador o de la impresora, lo que muestra que la informática y en especial el software educativo, adecuadamente construido y / o elegido como apoyo para las estrategias de enseñanza en el aula, funciona como un buen colaborador del profesor en su trabajo pedagógico. 
Otro aspecto de relevancia para que el software pueda motivar al alumno, y al mismo tiempo apoyar el proceso en su trabajo en el aula, son las estrategias integradas al software. En nuestro caso, el software "Hércules y Jiló", además de permitir una diversidad de exploración pedagógica por el profesor y por los alumnos, adoptó la estrategia de unir lo lúdico a lo educativo, para dejar al alumno libre para las adquisiciones que se planteaba y así favorecer aprendizajes significativos. Observamos muy claramente, en la aplicación del software, que esta combinación de lo lúdico como soporte para la acción educativa fue relevante para el trabajo pedagógico con los alumnos con deficiencia intelectual.

Interrogante 4 - ¿De qué manera el software educativo puede llevar al niño con deficiencia intelectual a asumir un papel activo en la adquisición de aprendizajes?

Observamos en su aplicación que las interfaces del software "Hércules y Jiló", las animaciones y el manejo de fácil comprensión han permitido que el alumno con deficiencia intelectual, el usuario al que está recomendado, jugó el papel activo en las actividades y juegos, favoreciendo la utilización de funciones cognitivas más sencillas en la perspectiva de aprendizaje.

Por otra parte, como plantearan los autores, el software se constituye en un ambiente multimedia y favorece la utilización de los más variados niveles de exploración lúdica y de aprendizaje. Los materiales constructivistas destacan la expresión y la exploración personales dando oportunidad a los alumnos para que desarrollen su propio aprendizaje, porque los consideran participantes activos en el proceso (VERDUGO, 1999).

Los alumnos con deficiencia intelecutal evolucionan en su desarrollo cognitivo si son estimulados a actuar con las personas y los objetos, siendo entonces capaces de elaborar sus propios conocimientos, como puede ser el lenguaje, el diseño, la dramatización, expresando una creatividad muy fecunda.

Interrogante $5-¿ Q u e ́$ elementos y estrategias puede presentar el software educativo para introducir al niño con deficiencia intelectual en una dinámica de adquisición de nuevos conocimientos, partiendo de conocimientos ya adquiridos?

La riqueza de recursos informatizados, de los contenidos (los seres vivos) y de estrategias, conforme se ha registrado en la fase de la investigación sobre la aplicación del software, está potenciado en el software "Hércules y Jiló" y es utilizado como recurso de apoyo al alumno y al profesor para atender a los diversos y diferentes niveles de interés y de adquisición de conocimiento.

La contextualización psicopedagógica, así como las orientaciones descritas que integran el software, permiten al profesor o tutor adecuar su aplicación para introducir nuevos conceptos y reforzarlos, siempre partiendo del nivel y de la necesidad y posibilidad del alumno. En la aplicación del software ante una clase especial para alumnos identificados con deficiencia intelectual que formó parte de esta investigación, se ha demostrado la riqueza de estrategias y posibilidades de enriquecimiento para el proceso de enseñanza-aprendizaje que pueden realizar estos alumnos.

Interrogante 6 - ¿Qué dinámica debe presentar el software educativo para garantizar al profesor un papel activo y mediador en el proceso de enseñanza- aprendizaje? 
La dinámica del software "Hércules y Jiló" por sí ya ofrece al profesor el papel activo como mediador de todo el proceso de aprendizaje. Los juegos y las actividades para realizar en el ordenador, imprimir, recortar, colorear, e instalar los juegos imponen una acción tanto para el alumno como para el profesor.

El profesor puede utilizar el software de varias maneras, porque se trata de un material didáctico realmente pensado y construido para permitir la exploración lúdico-pedagógica que el profesor quiera, incluso para explorar otros contenidos.

Lo que fue observado es que el software debe ofrecer la oportunidad de ser utilizado de varias maneras, así como favorecer el trabajo interdisciplinario.

\section{Interrogante $7-{ }_{i}$ De qué forma el software educativo puede funcionar como facilitador de la interrelación entre alumno-alumno, alumno-profesor, y alumno- conocimiento?}

La aplicación del software "Hércules y Jiló" ha demostrado que el software, con la estructura de juegos y posibilidades infinitas para la exploración pedagógica y que favorezca el trabajo colaborativo, puede constituir un excelente recurso pedagógico de apoyo al proceso de enseñanza-aprendizaje del alumno con deficiencia intelectual.

Todo esto es posible porque las situaciones creadas no separan al alumno del contexto de la sala de clase. Sitúa al niño en contacto con otros contenidos disponibles en el ambiente. Dispone de la presencia del profesor, de sus colegas y de los demás recursos que lo conducen a salir del aislamiento de un aprendizaje solitario para lograr un aprendizaje colectivo.

En el caso del software "Hércules y Jiló, los juegos no están limitados a su propia función ni al ambiente del ordenador: el alumno puede montarlos y jugar fuera del ordenador, así como los profesores pueden realizar otras actividades y explorar otros contenidos. Esto posibilita una variedad de exploración y puede dar oportunidad para que las interacciones acontezcan, una vez que los juegos atraen a los alumnos al trabajo colectivo.

Con esa dinámica, las interacciones proyectadas por el software "Hércules y Jilo" tratan al alumno como sujeto activo, afectivo y social que, a lo largo del proceso de construcción de conocimientos, construye también una auto-percepción y una autoestima, que conlleva a saberes colectivos (como los presentados por el software "Hércules y Jiló") y a saberes individuales (como los que él construye a partir de su propia experiencia como ser vivo e interactivo).

\section{CONCLUSIÓN}

Después de llevar a efecto realmente lo que aquí hemos expuesto, aplicarlo y reflexionar sobre ello, podemos afirmar que las Tecnologías de la Información y de la Comunicación, en particular el software educativo, están considerados como elementos de gran apoyo al trabajo del profesor y al proceso de aprendizaje del alumno.

Las TICs ofrecen una ayuda de gran importancia para la atención a las necesidades educativas especiales, que generalmente dificultan o impiden la integración del individuo 
en la escuela y en la sociedad. Enfatizamos la construcción de materiales como software educativos que, además de auxiliar el proceso de enseñanza-aprendizaje, puedan contemplar las singularidades de alumnos con necesidades educacionales especiales.

Estos materiales deben contener las potencialidades metodológicas-pedagógicas constructivas, un aspecto lúdico, posibilidades de intercomunicación, y que tecnológicamente sean amenos y amigables. Por todo esto y por el esmerado cuidado que se debe mimar en su elaboración tanto en sonido, como en imagen y en animación, pueden ayudar poderosamente a la integración social y escolar de los alumnos, como se ha demostrado en la investigación realizada con el software educativo "Hércules y Jiló".

\section{Notas}

1. El software" Hércules y Jiló" es recomendado para alumnos con deficiencia intelectual que se encuentran en el nivel de aprendizaje que corresponde a las dos primeras series/años de estudios del primer ciclo de la Enseñanza Fundamental.

2. La AAMR (Asociación Americana sobre Retardo Mental) define en 1992: "El retraso mental (deficiencia intelectual) hace referencia a limitaciones sustanciales en el desenvolvimiento corriente. Se caracteriza por un funcionamiento intelectual significativamente inferior a la media, que tiene lugar junto a limitaciones asociadas en dos o más de las siguientes áreas de habilidades cognitivas posibles: comunicación, cuidado personal, vida en el hogar, habilidades sociales, utilización de la comunidad, auto - gobierno, salud y seguridad, habilidades académicas funcionales, ocio y trabajo. El retraso mental se manifiesta antes de los dieciocho años".

\section{Referencias}

ALMEIDA, Maria Elizabeth Bianconcini. Informática e formação de professores. Brasília: Proinfo / MEC / SEE, 2001.

ALONSO, Catalina Maria. Aprendizaje y ordenador. Madrid: Dykinson, 2001.

COSCARELLI, Carla Viana. Leitura em ambiente multimedia e a produção de inferências. 1999. 320f. Tese (doutorado), Faculdade de Letras. UFMG, Belo Horizonte.

ESPAÑA, MEC / ES. Nuevas tecnologías y necesidades educativas especiales: orientaciones para la atención a la diversidad. Vol. 1. Guía al profesor y otros implicados en el proceso de enseñanza /aprendizaje, a la hora de utilizar los medios informáticos como recurso educativo con el alumno con necesidades educativas especiales. Madrid: MEC, 1992.

GRINSPUN, Mirian Paura Sabrosa Zippin. Educação tecnológica: desafios e perspectivas. São Paulo: Cortez, 1999.

LÉVY, Pierry. A conexão planetária. Rio de Janeiro: Editora 34, 2001.

LÓPEZ, Maria José. Nuevas tecnologias de la información y comunicación para la educación. Sevilla: Alfar, 1995.

MARTÍ, Eduardo. Aprender con el ordenador en la escuela. Barcelona: Ediciones de la Universidad de Barcelona; ICE -Horsori, 1992. 
PAPERT, Seymour. La máquina de los niños. Barcelona: Piados, 1995.

. Logo: computadores e educação. Trad.: José Armando Valente; Beatriz Bitelman e Afira Vianna Ripper. São Paulo: Brasiliense, 1981.

PÉREZ, Isabel Paula. Educación especial: técnicas de intervención. Madrid: Mcgraw Hill, 2003.

PRIETO, Maria Dolores Sánchez Variables del profesor como mediador del proceso instruccional. In: BELTRÁN, Jesús; GENOVARD, Cándido (Eds.), Psicología de la instrucción I. Variables y procesos básicos. Madrid: Síntesis. 1996. p. 194-216.

SANTOS, Gilberto Lacerda. Proposta de uma estratégia holística para a engenharia de softwares educativos. Revista Brasileira de Tecnologia Educativa, Brasília, n. 148, p. 22-26, 2000.

SCOZ, Beltran. Psicopedagogía e realidade escolar: o problema escolar e de aprendizagem. Petrópolis: Vozes. 1994.

SILVA, Marco. Sala de aula interativa. 2. ed. Rio de Janeiro: Quartet, 2004.

SKINNER, Burrhus Frederick. Análise do comportamento. São Paulo: EPU, 1973.

SOUZA, Amaralina Miranda. Desarrollo de un ambiente de aprendizaje de carácter cooperativo destinado al alumno con deficiencia intelectual: el software "Hércules y Jiló". Anales del XVII Congreso Internacional de Informática Educativa 2005--Madrid: Uned - España, 2005.

; SANTOS, Gilberto Lacerda. "Hércules y Jiló”: um software educativo de apoio á formação docente para atuar junto a crianças com deficiência mental. Linhas Críticas. Brasília, v. 7, n. 13, p. 275-288, jul.-dez. 2001.

TARDIF, Jacques Por un enseignement strategique: l'apport de la psichologie cognitive. Quebec: Logiques, 1992.

TORRES, Sánchez. La palabra complementada. Madrid: Cepe, 1990.

VALENTE, José Armando. Aprendendo com o computador. Campinas: Biblioteca Central Unicamp, 2001.

1991.

. Liberando a mente: computadores na educação especial. São Paulo: Biblioteca Central,

VERDUGO Miguel Angel. Avances conceptuales actuales y del futuro inmediato: revisión de la definición de 1992 de la AAMR. Revista Siglo Cero, Madrid, v. 30, n. 5, p. 27-31, 1999.

. Un nuevo concepto de retraso mental. Salamanca: Ediciones de la Universidad de Salamanca, 1997.

VIGOTSKI, Liev Semionovitch. A formação social da mente. Titulo original: Mind in society. Trad.: José Cipolla Neto, Luis Silveira Menna Barreto, Solange Castro Afeche. São Paulo: Martins Fontes, 2000.

Aprendizado e desenvolvimento: um processo sócio-histórico. Trad.: Marta Kohl de

Oliveira. Porto Alegre: Artmed, 1994. 


\title{
As tecnologias aplicadas à educação especial integradora: a contribuição do software educativo "Hércules e Jiló" Resumo
}

As Tecnologias de Informação e de Comunicação aparecem como grandes aliadas do professor, favorecendo as diferentes formas de aprender e as diferenças individuais dos alunos e dos professores. Apresentamos as linhas fundamentais que servem de base no desenho, na elaboração e na explicação do software educativo "Hércules e Jilo", recomendado para o trabalho pedagógico com alunos que apresentam necessidades educativas especiais. Neste trabalho serão abordadas várias linhas de ação: 1. fundamentar sua realização com base cientifica e tecnológica para lograr uma relação e motivação amigável do aluno com a máquina; 2. oferecer uma base científico-pedagógico-metodológica para tentar alcançar atrativos os quais $o$ aluno possa construir conhecimento pela sua interação com o software e suas atividades derivadas. Aspira-se que as tecnologias - em particular, o software educativo - contribuam na aprendizagem de alunos que apresentam necessidades educativas especiais ou não.

Palavras-chave: Tecnologias da Informação e da Comunicação. Software educativo. Educação especial.

\section{The Technologies of Information and Communication Applied to Integrating Special Education: the contribution of the educational software "Hércules and Jiló"} Abstract

Information and Communication Technologies become great allies of the teacher in order to facilitate the different forms of learning and favor the individual differences of students and teachers. We present the experience of creating and applying the educational software "Hércules y Jilo" recommended for a pedagogical relationship with pupils with special educational needs. In this process, several courses of action have been dealt with: 1 . base its realization on a scientific and technological basis so as to reach a friendly relationship and motivation of the student with regards to the equipment; 2. offer a scientific, pedagogical, methodological foundation to try to become an attraction such that the student can construct knowledge through his interaction with the software and related activities. We believe that technologies in general and the educational software in particular can contribute towards the learning process of all students, whether they have special educational needs or not.

Keywords: Information and Communication Technologies. Educational Software. Special Education.

\section{Les Technologies de l'Information et de la Communication Appliquées à l'Éducation Spéciale Intégrative : la contribution du logiciel éducatif "Hércules et Jiló"}

\section{Résumé}

Les technologies de l'information et de la communication deviennent de grands alliés pour le maître pour faciliter les différentes formes d'apprendre et les différences individuelles des élèves et maîtres. Nous présentons l'expérience de créer et appliquer le logiciel éducatif "Hércules et Jiló" recommandé en vue d'une relation pédagogique avec les élèves porteurs de besoins spéciaux. Selon ce processus, plusieurs cours d'action ont été pris : 1 . donner à la réalisation une base scientifique et technologique de manière à ce que l'on arrive à une relation amicale et à une bonne motivation de l'élève avec l'équipement; 2. offrir un fondement scientifique, pédagogique et méthodologique pour que l'élève sente un attrait pour construire son savoir en interaction avec le logiciel et activités dérivées. Nous croyons que les technologies en général et le logiciel éducatif en particulier peuvent contribuer à l'apprentissage de tous les élèves, qu'ils soient porteurs de besoins spéciaux ou non.

Mots clefs : Technologies de l'Information et Communication. Logiciel Éducatif. Éducation Spéciale.

\author{
Recebidas I ${ }^{a}$ versão em: 22.02.2007 \\ $2^{a}$ versão em: 08.08.2007 \\ Aceita $3^{\mathrm{a}}$ versão em: 10.09.2007
}

